\title{
Avatar Therapy Revised.
}

Dr Bheemaiah, Anil Kumar, Vayu Vaidya, Seattle WA, 98125 WA, USA.

miyawaki@yopmail.com

Abstract:

This paper describes a revision of the Avatar Therapy, also known in the published literature as the Computer Voice Assisted Therapy. A Plushie based version of the therapy is described with two innovations from Vayu Vaidya, a R\&D organization directed by the author, Pillow-Buddies ( $\left.{ }^{T M}\right)$ and Cuddles( ${ }^{\text {TM}}$ ). The use of AWS Polly for timbre and pitch based control of the computer assisted voice is described.

A framework for describing the positification and optimism templates for conversational UI design is described, based on the C+ language.

Keywords: C+ Language, positification template, optimism template, AWS Polly, Avatar Therapy, Computer voice assisted therapy, conversational UI, Amazon Alexa Skills, Infotainment, Vayu Vaidya.

What:

Avatar therapy has failed, in FDA approval or in the form of on-screen based avatar, Since people relate to material objects, the customized cartoon to plushies software service by Vayu Vaidya allows you to sketch an avatar and customize the 'voice' in the Plushie, using Polly or Lex and choose standard template responses for Positification and Optimism. (See Bheemaiah 2019, See-Positive or C+)

How: 
Cartoons are drawn by the patient and converted to polishes with an embedded Bluetooth speaker. This connects to any device with a browser that can download JavaScript files to run a lex based conversational UI with Positification templates. The Lex UI also has templates for optimism and mood molding, to uplift your emotional makeup similar to Replika.ai.

So What:

Clinical Trials are the next step in proving the efficacy of revised avatar therapy, I an a companion paper I describe, my clinical experiences with the revised avatar therapy both in imaginary friends as iconized by the cartoon series "Calvin and Hobbes", and in mitigation of threats and negative voices in both the workplace and home, using revised avatar therapy for avatar dissolution.(Mayor 2017; T. Craig, Ward, and Rus-Calafell 2017; T. J. Craig, n.d.; Stefaniak et al. 2019)

\section{Introduction.}

A decades experience in research psychiatric roles in conjunction with advocacy roles, has led me to be a proponent of a differently-abled view of a psychosis spectrum disorder, while hearing voices has been reported to be quite a normal experience in literature, many have even attributed, social or scientific and even spiritual success owing to inner voice guidance.

In some cases, voices have been even attributed to an over-inflated cost of living and the inability to afford communication services like landlines and cellphones.

Only in extreme cases of aggression based on auditory hallucinations, or extreme psychosis induced by hallucinations is intervention prescribed, with Avatar Therapy being one of the leading synthesized voice-assisted therapies. While traditional avatar therapy has used a monitor based visual indication of an avatar, with patient-controlled voice parameters, a one to one correspondence with the hallucinations can lead to unwanted side effects like monitor based sickness and other symptoms.

A deviation from this therapy using Plushies, both active and passive has been found to be more effective with fewer side effects, given the natural association of voices and identities with Plushies in children. In this paper, we investigate scientifically if this correlation of 'imaginary friendship' with Plushie based identities, can be used in the context of Avatar Therapy. We then investigate the role of active synthetic voices in these Plushies, using embedded Bluetooth speakers and Amazon Echo sets, with specially developed conversational UI, using AWS Lex and Google based Replika.ai.

\section{Discussion.}

While traditional avatar therapy uses the services of a psychiatrist to control and voice an avatar on-screen, to use a principle of induction, to control and dissolve internal voices in a patient, the altered Plushie based therapy involves the patient first cartooning the 
perceived avatar or inner voice and building a Plushie with that cartoon using an automated service provided by Vayu Vaidya, a research center directed by the author.

Self-correction of the inner voice is induced by speaking with an imaginary friend in the Plushie, till the corresponding inner voices fade out. This is the passive plushie approach which can be with or without psychiatric help.

An active approach similar to the traditional therapy involves the use of the Pillow Buddies $\left({ }^{\mathrm{TM}}\right)$ or Cuddles $\left({ }^{\mathrm{TM}}\right)$ product, where two templates, positification, and optimism are used in Lex's responses with psychiatric help in a CBT and MiCBT framework built into the conversational UI.

Replika.ai, already provides a number of such conversational UI based voice services, which can be easily integrated with the plushie. This provides induction based positive which provides mood upliftment and suggestive help with mood disorders, depression, seasonal affective disorders, borderline bipolar and other treatment-resistant psychosis spectrum disorders.

\section{Infotainment Based Approach.}

Infotainment, is information plus

entertainment, predictive modelling is a bio-statistical approach to wellness and risk mitigation through a wellness therapy, with or without prescription medicines. It can also form integrative and alternative therapies in the case of mental wellness. Vayu Vaidya, a R\&D organization, directed by the author publishes infotainment on various topics, including Avatar Therapy.
An Alexa Skill is published which can be subscribed to.("Amazon.com: Avatar Therapy By Vayu Vaidya: Alexa Skills" n.d.)

\section{Pillow Buddies( ${ }^{\mathrm{TM}}$ ) and Cuddles( ${ }^{\mathrm{TM}}$ ) from Vayu Vaidya, passive plushies vs active plushies.}

Passive plushies are either passive plushie toys or toys created from images, with or without thermoregulation like the Cuddles ${ }^{\text {TM }}{ }^{\mathrm{M}}$. While the world of plushie based toys offers a channel for avatar therapy, not restricting the mimicking of the avatar's voice by timbre and pitch, active plushies, based on the Pillow Buddies $\left({ }^{\mathrm{TM}}\right)$ with computer generated voice therapy as avatar therapy have a timbre and pitch control, like the web speech(Smus 2013) API of html5 and that of Amazon Polly("Amazon Polly" n.d.) or Azure("Cognitive Speech Services | Microsoft Azure" n.d.).

\section{Positification as a Template}

\section{Response.}

$\mathrm{C}+$ or See Positive is a new language of semantics and object oriented programming, that bridges the machine language world with higher level provable constructs with code recycling and automatic code generators. It is also a vehicle for spirituality, a transformational medicine of positification and optimism endemic to the indigenous communities of the planet, especially the Native Americans and the Himalayan and Tibetian people. A higher happiness index.

A template for positification can be described formally as an extension to a request-response architecture, based on models of natural language, which have evolved from AIML based 
state machines to a more comprehensive understanding of intents, sentiments and semantics, now part of NLP, conversational modeling techniques have improved with meta data models, persistence and the simulation of understanding, emotional intelligence and consciousness and learning, as relevant to present day conversational UI.

Template responses are one form of programming in conversational UI("Resources | Conversational UI Tools" n.d.). Here we describe the use of positification as defined in $\mathrm{C}+$ language, for this purpose.

Templates, in conversational UI design consist of personality and response templates, similar to chat bot templates, which you can fork and enhance(Bheemaiah, n.d.)concepts are with Google actions and Google dialog flow designs, where pre-trained templates can be added.(Bheemaiah, n.d.; Biswas 2018) this publication, the author introduces, yet another template definition, a conversation filter template, a design of a template pattern for positification of request-response in voice design.

Positification is defined as the transformation of any mechanism defined by a set of actions into something that works for you to an end that is useful to you in value addition.

if there exist a set of actions [A], defined in the context of a conversational model or a conversational UI, positification() is defined as an inheritable cloud function that maps $[\mathrm{A}]$ to [PA], a set of actions with necessary positive side effects.

There may not always exist a complete mapping for every action $A$ in $[A]$ to $[P A]$ and hence a subset of actions can be mapped to a positified action. if there exists any action $A$ in $[A]$ with a non positive value, quantified by a measurable likeness function defined on that action,

then, there is a search to map A to PA, an action with the same inputs with a positive value as defined by the likeness function. The conversion from $A$ to $P A$ is called positification.

Future work will define a complete set of heuristics for the A to PA conversion, and polynomial time algorithms for decidability on the transformation.

\section{Optimism as a Template Response.}

Similar to positification, is the optimism template response to be described by the optimization() function, for a, A to OA transformation of non optimistic actions, optimism is thus a re-interpretation of value addition by actions, where the outcome or value addition is not deterministic.

\section{Conclusions and Future Work.}

The author has thus presented, in the language of positification, the language of $\mathrm{C}+$ or See-positive, an objective OOPS language, the existence and definitions of positification and optimism, as defined in H.H Dalai Lama and Bishop Tutu's book on the Joys of Living(Lama, Tutu, and Abrams 2016; Lama and Tutu 2016). Thus we prove the vacation hypothesis(Bheemaiah n.d.) and the increased happiness index in a revision of the avatar therapy.

The avatar therapy is enhanced, rendered safer in practice with the use of plushies, and two technologies, the smart pillow with Alexa 
integration called Pillow Buddies $\left({ }^{\mathrm{TM}}\right)$ and the thermo regulation pillow called Cuddles $\left.{ }^{\mathrm{TM}}{ }^{\mathrm{M}}\right)$. The existence of physical plushies, rendered in the world of stuffed toys, stuffed with positive knowledge and made programmable, would be a stronger avatar based therapy.

Future work involves a heuristic framework for optimistic and positification templates.

\section{References.}

"Amazon.com: Avatar Therapy By Vayu Vaidya: Alexa Skills." n.d. Accessed September 26, 2019.

https://www.amazon.com/Avatar-Therapy -By-Vayu-Vaidya/dp/B07QBBHD68/ref=sr 1_1 ?keywords=vayu+vaidya\&qid=1569512 $882 \& s r=8-1$.

"Amazon Polly." n.d. Amazon Web Services, Inc. Accessed September 26, 2019. https://aws.amazon.com/polly/.

Bheemaiah, Anil Kumar. n.d. "Computational Intelligence in Conversational UI, A BotLibre Case Study. A Survey Paper." https://doi.org/10.31224/osf.io/2ybpq.

- - . n.d. "Theological Fundamentalism: A Personal Experience." Accessed September 26, 2019. https://www.academia.edu/40424573/The ological_Fundamentalism_A_Personal_Exp erience.

Biswas, Manisha. 2018. "Wit.ai and Dialogflow." Beginning Al Bot Frameworks. https://doi.org/10.1007/978-1-4842-37540_3.

"Cognitive Speech Services | Microsoft Azure." n.d. Accessed September 26, 2019. https://azure.microsoft.com/en-us/service s/cognitive-services/speech-services/.

Craig, Thomas Jamieson. n.d. "Reducing the Frequency and Severity of Auditory Hallucinations: The AVATAR Clinical Trial." Http://isrctn.org/>. https://doi.org/10.1186/isrctn65314790.

Craig, Tom, Tom Ward, and Mar Rus-Calafell. 2017. “Erratum to: AVATAR Therapy for
Refractory Auditory Hallucinations." Brief Interventions for Psychosis.

https://doi.org/10.1007/978-3-319-305219_14.

Lama, Dalai, and Desmond Tutu. 2016. The Book of Joy. Random House.

Lama, Dalai, Desmond Tutu, and Douglas Carlton Abrams. 2016. The Book of Joy: Lasting Happiness in a Changing World. Penguin.

Mayor, Susan. 2017. "Avatar Therapy Reduces Auditory Hallucinations in Schizophrenia, Trial Finds." BMJ. https://doi.org/10.1136/bmj.j5458.

"Resources | Conversational UI Tools." n.d. Conversational UI Tools. Accessed September 26, 2019. https://cui.tools/resources/.

Smus, Boris. 2013. Web Audio API. "O’Reilly Media, Inc."

Stefaniak, Izabela, Kamil Sorokosz, Artur Janicki, and Jacek Wciórka. 2019. "Therapy Based on Avatar-Therapist Synergy for Patients with Chronic Auditory Hallucinations: A Pilot Study." Schizophrenia Research. https://doi.org/10.1016/j.schres.2019.05.0 36. 